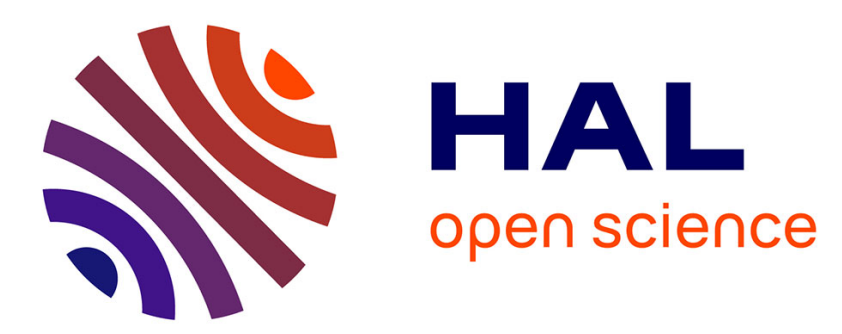

\title{
Robust and Adaptive Active Vibration Control Using an Inertial Actuator
}

\author{
Tudor-Bogdan Airimitoaie, Ioan Doré Landau
}

\section{To cite this version:}

Tudor-Bogdan Airimitoaie, Ioan Doré Landau. Robust and Adaptive Active Vibration Control Using an Inertial Actuator. IEEE Transactions on Industrial Electronics, 2016, 63 (10), pp.6482-6489. 10.1109/TIE.2016.2548438 . hal-01394199

\section{HAL Id: hal-01394199 \\ https://hal.science/hal-01394199}

Submitted on 18 Nov 2016

HAL is a multi-disciplinary open access archive for the deposit and dissemination of scientific research documents, whether they are published or not. The documents may come from teaching and research institutions in France or abroad, or from public or private research centers.
L'archive ouverte pluridisciplinaire HAL, est destinée au dépôt et à la diffusion de documents scientifiques de niveau recherche, publiés ou non, émanant des établissements d'enseignement et de recherche français ou étrangers, des laboratoires publics ou privés. 


\title{
Robust and Adaptive Active Vibration Control Using an Inertial Actuator
}

\author{
Tudor-Bogdan Airimitoaie and Ioan Doré Landau
}

\begin{abstract}
An active vibration control system using an inertial actuator will be used to assure the vibration isolation of a chassis in the presence of multiple unknown time varying tonal vibrations located in two distinct regions in the frequency domain. The objective is to minimize the residual force by applying an appropriate control effort through the inertial actuator. The system does not use any additional sensor for getting in realtime information upon the disturbances. A hierarchical feedback approach will be used for the control of the system. At the first level a robust linear controller will be designed taking advantage of the knowledge of the domains of variation of the frequencies of the vibrations. To further improve the performance, a direct adaptive control algorithm will be added. Its design takes into account the internal model principle for disturbance rejection and is conveniently implemented through the Youla-Kučera parametrization of the controller. Experimental results obtained on a relevant test bench will illustrate the methodology.
\end{abstract}

Index Terms - Adaptive regulation, system identification, active vibration control, robust control, Youla-Kučera parametrization, internal model principle.

\section{Introduction}

A CTIVE VIBRATION CONTROL (AVC) is a current challenge in todays technology development for smart vibration isolation systems. Fundamentally in AVC a compensator system is introduced with the aim to generate a secondary "source." This compensator conveniently driven will interfere destructively with the vibrations coming from the original source (in general non accessible).

The technology for the implementation of the AVC in mechanical structures has evolved towards using inertial (electrodynamic) actuators [1]. In this paper we will consider the case of AVC systems using inertial actuators in a co-located configuration "actuator-sensor."

Vibrations in terms of control terminology are "disturbances" which have to be compensated (see also [2], [3]). They can be conveniently described in the frequency domain. One encounters either "tonal" (sinusoidal) disturbances with unknown and time varying frequency or "narrow band disturbances" located in a certain frequency region.

Initial solutions for this problem have been based on "feedforward" compensation techniques, provided that an "image" (called also "reference") of the disturbance can be obtained by using an additional sensor [4]. This approach has a number of disadvantages: (i) it requires the use of an

T.-B. Airimitoaie is with the Univ. Bordeaux, IMS, UMR 5218, F-33405 Talence, France (e-mail: see http://tudor-bogdan.airimitoaie.name/).

I.D. Landau is with the CNRS, GIPSA-lab, UMR 5216, F-38402 Saint Martin D'Hères, France (e-mail: ioan-dore.landau@gipsa-lab.grenoble-inp.fr).

Manuscript received October 9, 2015; revised October 10, 2015 and February 2, 2016; accepted March 1, 2016. additional transducer, (ii) difficult choice for the location of this additional transducer, (iii) requires adaptation of many parameters, and (iv) presence of a physical internal "positive" feedback coupling (a challenging stability issue). In fact, in $\mathrm{AVC}$, in order to attenuate narrow band disturbances and tonal disturbances, a feedback control structure can be used.

In managing the vibration attenuation by feedback, the shape of the modulus of the "output sensitivity function" (the transfer function between the disturbance and the residual acceleration/force) is fundamental both from performance and robustness considerations. Three basic concepts are to be considered: the Bode Integral, the Modulus margin and the Internal Model Principle (IMP). The Bode Integral just says that the area of attenuation in the frequency domain (attenuation $\times$ frequency range) leads to an amplification at other frequencies such that the integral of the modulus of the sensitivity function over the frequency domain is equal to zero. The modulus margin, which is the inverse of the maximum of the modulus of the output sensitivity function, gives the minimum distance with respect the the Nyquist instability point and as such is a major indicator both for robustness and performance. The IMP says that in order to cancel completely a disturbance the controller should contain the model of the disturbance $^{1}$ (see also [5]).

Several problems have been considered in the field of AVC. The case of full rejection of single or multiple tonal disturbances (up to 3) located quite distantly in the frequency domain with unknown and time varying frequencies over a significant frequency range has been extensively covered in the literature and compared experimentally [6]. ${ }^{2}$ An adaptive feedback approach taking advantage of the IMP as well as of a special parametrization of the controller (the Youla-Kučera parametrization [7]) has been considered. Note that YoulaKučera parametrization introduces explicitely a distrubance observer. A rapprochement with DOB control method [8] can be established.

In this paper, a different pertinent problem encountered in practice will be examined. One considers multiple unknown and time varying sinusoidal disturbances located within two distinct relatively small frequency ranges. To be specific, two cases will be considered: (i) the case of two time varying tonal disturbances located in two distinct frequency regions and (ii)

\footnotetext{
${ }^{1}$ In the case of a single "tonal" disturbance (for a pure single frequency vibration), this corresponds to a zero damped second order tuned at this frequency. The gain of the output sensitivity function at this frequency will be $0(-\infty \mathrm{dB})$.

${ }^{2}$ More details can be found on the website http://www.gipsa-lab. grenoble-inp.fr/ ioandore.landau/benchmark_adaptive_regulation/
} 
the case of four simultaneous tonal disturbances, two located in one limited frequency range and the other two in another frequency range.

In this context, a very important problem is to be able to counteract the very low frequency oscillations which are generated when the two frequencies are very close. This phenomenon is considered for example in [9]. It also occurs often on ships with two thrusters which can not be perfectly synchronized. A typical image of the phenomenon is shown in Figure 1.

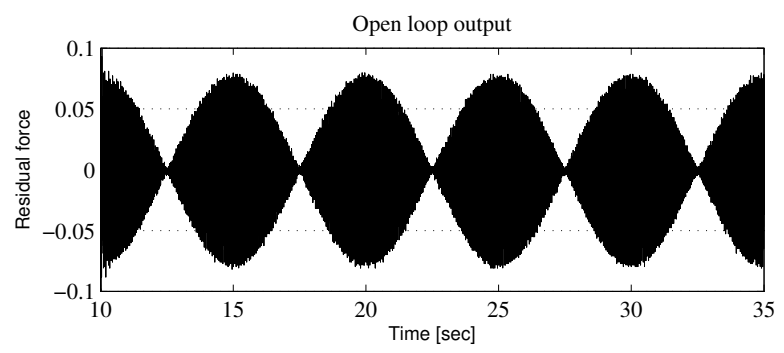

Figure 1. Vibrational interference caused by two sinusoidal disturbances with close frequencies.

Since these disturbances are located within two relatively small frequency ranges, it is possible to consider a robust linear control design which will shape the output sensitivity function in such a way that a sufficient attenuation is introduced in these two frequency regions but avoiding significant amplification at other frequencies (both for performance and robustness reason). This problem in the context of active noise control has been considered in [10] and the shaping of the output sensitivity function has been achieved using the convex optimization procedure introduced in [11]. It will be shown in this paper that an elementary procedure for shaping appropriately the modulus of the sensitivity functions can be implemented using stop band filters as shaping tools. For a basic reference on this approach see [12].

To further improve the performance, an algorithm for direct adaptive rejection of the disturbances will be added [13].

Another important point of the methodology for designing AVC systems is the fact that one uses for design discrete-time models of the system directly estimated from data (both the orders of the model and the parameters). The motivation is two fold: on one hand it is practically impossible to obtain an accurate model of the plant from physical modeling based on continuous time equations and on the other hand very performant methods for estimating orders and parameters of discrete time dynamical models directly from data are available. Furthermore designing the control law based on the identified discrete time model will directly provide the control algorithm to be implemented on the computer controlling the system.

The paper is organized as follows. In section II, the test bench and its characteristics will be presented. Section III presents briefly the equations describing the system model and the controller. Section IV presents the performances specification to be achieved and the design of the robust controller. Section V presents the adaptive control algorithm. Experimental results for both robust linear control and adap- tive control are given in Section VI. Concluding remarks are given in Section VII.

\section{System Presentation}

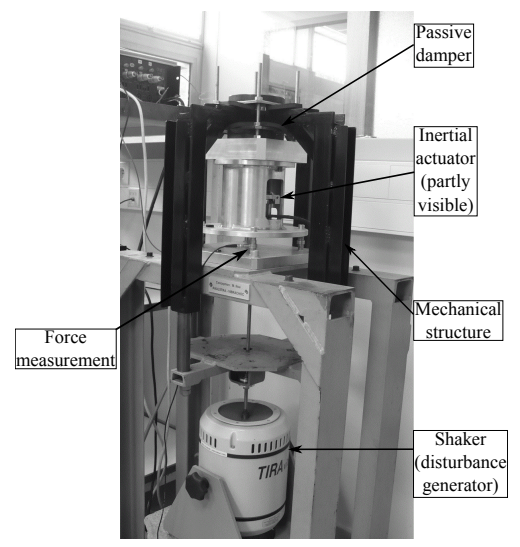

Figure 2. The active vibration control system (photo).

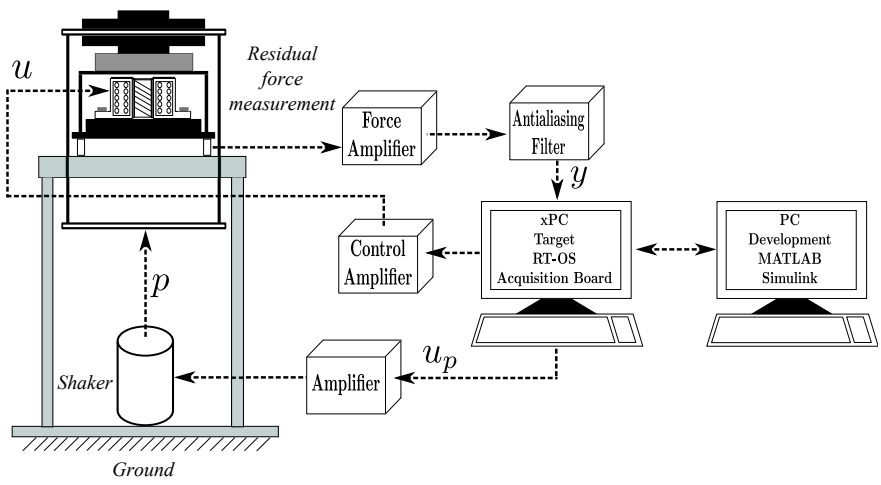

Figure 3. The active vibration control data acquisition diagram.

The AVC system that will be used for the experiments is shown in Fig. 2. The same system has been used for an international benchmark on adaptive disturbance regulation (see [6]).The system has been designed in order to test the performance of active control by reducing to a minimum the "passive vibration attenuation". It consists of a shaker (fixed to the ground), a passive damper, an inertial actuator, a mechanical structure, and a transducer for the residual force. For control purposes, 2 desktop computers are used, one with a Microsoft Windows operating system and Matlab/Simulink environment (for controller design, implementation and simulation) and the second with the real time operating system Matlab xPC Target (for real-time operation of the AVC system) - see also Fig. 3 for more details. The latter is connected through a power amplifier to the inertial actuator.

The mechanical construction is such that the vibrations produced by the shaker are transmitted to the upper part of the mechanical structure, on top of the passive damper. The inertial actuator is fixed to the chassis where the vibrations should be attenuated. The controller, through the power amplifier, generates current in the coil which produces motion in order to reduce the residual force. In Fig. 3, the control signal $u(t)$ represents the position of the magnet inside the inertial actuator. The measured output of the system (residual 
force) is $y(t)$ which enters the xPC Target dedicated computer. Finally, for testing purposes, the disturbance $p(t)$ induced by the shaker on the residual force is operated from the computer through the disturbance input $u_{p}(t)$. The transfer function between the disturbance input $u_{p}(t)$ and the measured output $y(t)$ is called primary path. The transfer function between the control input $u(t)$ and the measured output is called secondary path. Note that the system has a double differentiator behavior (input=position, output=force).

The physical parameters of the system being unknown, black-box discrete time linear model identification has to be done in order to obtain a dynamical model of the primary and secondary paths. ${ }^{3}$ The sampling period is $T_{s}=0.00125 \mathrm{sec}$ $\left(f_{s}=800 \mathrm{~Hz}\right)$.

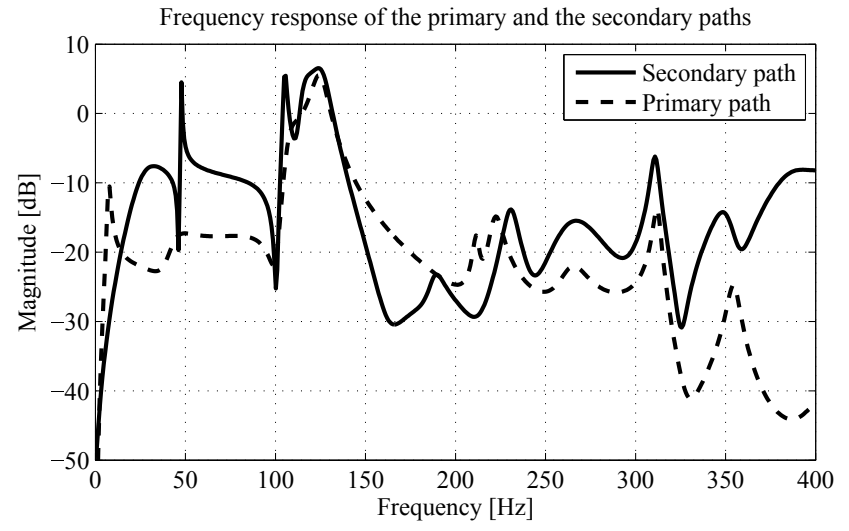

Figure 4. Frequency characteristics of the secondary and the primary paths.

Fig. 4 gives the frequency characteristics of the identified model for the primary and secondary paths. As it can be seen, there is an important number of very low damped complex poles (resonances) and complex zeros (anti-resonances). The primary path model is used only for simulation purposes. The region for active vibration control is 50 to $95 \mathrm{~Hz}$.

\section{System Description}

The linear time invariant (LTI) discrete time model of the secondary path, used for controller design is

$$
G\left(z^{-1}\right)=\frac{z^{-d} B\left(z^{-1}\right)}{A\left(z^{-1}\right)}=z^{-d} \frac{1+a_{1} z^{-1}+\cdots+a_{n_{A}} z^{-n_{A}}}{b_{1} z^{-1}+\cdots+b_{n_{B}} z^{-n_{B}}}
$$

and $d$ is the plant pure time delay in number of sampling periods. ${ }^{4}$

The output of the plant $y(t)$ and the input $u(t)$ may be written as (see Fig. 5):

$$
\begin{aligned}
y(t) & =\frac{q^{-d} B\left(q^{-1}\right)}{A\left(q^{-1}\right)} \cdot u(t)+p(t), \\
S_{0}\left(q^{-1}\right) \cdot u(t) & =-R_{0}\left(q^{-1}\right) \cdot y(t) .
\end{aligned}
$$

In (2), $p(t)$ is the effect of the disturbances on the measured output ${ }^{5}$ and $R_{0}\left(z^{-1}\right), S_{0}\left(z^{-1}\right)$ are polynomials in $z^{-1}$ having

\footnotetext{
${ }^{3}$ Both the orders and the parameters of the models have been estimated from data.

${ }^{4}$ The complex variable $z^{-1}$ will be used to characterize the system's behavior in the frequency domain and the delay operator $q^{-1}$ will be used for the time domain analysis.

${ }^{5}$ The disturbance passes through a so called primary path which is not represented in this figure, and $p(t)$ is its output.
}

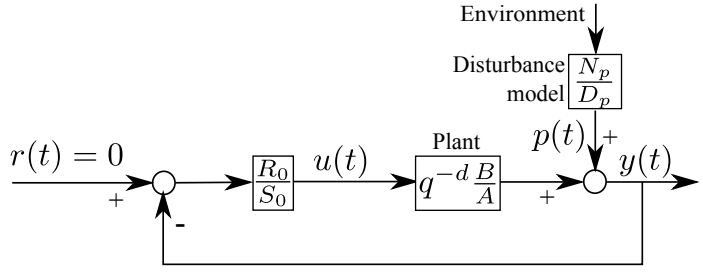

Figure 5. Feedback regulation scheme for rejection of disturbances.

the following expressions: ${ }^{6}$

$$
\begin{aligned}
& S_{0}=1+s_{1}^{0} z^{-1}+\ldots+s_{n_{S}}^{0} z^{-n_{S}}=S_{0}^{\prime} \cdot H_{S_{0}}, \\
& R_{0}=r_{0}^{0}+r_{1}^{0} z^{-1}+\ldots+r_{n_{R}}^{0} z^{-n_{R}}=R_{0}^{\prime} \cdot H_{R_{0}},
\end{aligned}
$$

where $H_{S_{0}}\left(z^{-1}\right)$ and $H_{R_{0}}\left(z^{-1}\right)$ represent pre-specified parts of the controller (used for example to incorporate the internal model of a disturbance or to open the loop at certain frequencies) and $S_{0}^{\prime}\left(z^{-1}\right)$ and $R_{0}^{\prime}\left(z^{-1}\right)$ are the solutions of the Bezout equation

$$
P_{0}=\left(A \cdot H_{S_{0}}\right) \cdot S_{0}^{\prime}+\left(z^{-d} B \cdot H_{R_{0}}\right) \cdot R_{0}^{\prime} .
$$

In the last equation, $P_{0}\left(z^{-1}\right)$ represents the characteristic polynomial, which specifies the desired closed loop poles of the system.

The transfer functions between the disturbance $p(t)$ and the output of the system $y(t)$ and from disturbance to the control input $u(t)$, denoted respectively output sensitivity function and input sensitivity function, are given by

$$
S_{y p}\left(z^{-1}\right)=\frac{A\left(z^{-1}\right) S_{0}\left(z^{-1}\right)}{P_{0}\left(z^{-1}\right)}
$$

and

$$
S_{u p}\left(z^{-1}\right)=-\frac{A\left(z^{-1}\right) R_{0}\left(z^{-1}\right)}{P_{0}\left(z^{-1}\right)} .
$$

It is important to remark that one should only reject disturbances located in frequency regions where the plant model has enough gain. This rule results from (7) and noticing that perfect rejection at a certain frequency $\omega_{0}$ is obtained iff $S_{0}\left(e^{-j \omega_{0}}\right)=0$. At this frequency, under perfect rejection of disturbances, one gets

$$
S_{u p}\left(e^{-j \omega_{0}}\right)=-\frac{A R_{0}}{0+e^{-d j \omega_{0} B R_{0}}}=-\frac{A}{e^{-d j \omega_{0} B}}=\frac{1}{G\left(e^{-j \omega_{0}}\right)} .
$$

Eq. (9) corresponds to the inverse of the gain of the system to be controlled. Its implication is that cancellation (or in general an important attenuation) of disturbances on the output should be done only in frequency regions where the system gain is large enough. If the gain of the controlled system is too low, $\left|S_{u p}\right|$ will be large at these frequencies. Therefore, the robustness vs additive plant model uncertainties will be reduced and the stress on the actuator will become important [12]. Eq. (9) also implies that serious problems will occur if $B\left(z^{-1}\right)$ has complex zeros close to the unit circle (stable or unstable zeros) at frequencies where an important attenuation of disturbances is required. It is mandatory to avoid attenuation of disturbances at these frequencies.

\footnotetext{
${ }^{6}$ The argument $\left(z^{-1}\right)$ will be omitted in some of the following equations to make them more compact.
} 


\section{Robust Control Design}

Before presenting the objectives for regulation and robustness, a few notions about feedback disturbance attenuation should be reminded. In the case of a feedback controlled system, the Bode integral constraint leads to a waterbed effect on the output sensitivity function (transfer function from disturbance $p(t)$ to output $y(t)$ in closed loop, see Section III). In other words, forcing the magnitude of the output sensitivity function at certain frequencies below $0 \mathrm{~dB}$ (in order to attenuate disturbances) has an inverse effect at neighboring frequencies, where an amplification will be observed. Recalling from [12] that the minimal distance between the Nyquist plot of the open loop transfer function and the critical point $-1+0 i$ (also called modulus margin) corresponds to the inverse of the maximum of the output sensitivity function, it can be concluded that "too much" attenuation at some frequencies can have a bad effect on the robust stability of the closed loop system.

The secondary path has enough gain in the frequency region of operation (see also Fig. 4).

For the design of the linear robust digital controller the following specifications are considered:

- up to 4 sines disturbances are supposed to affect the output of the system

- their frequencies are varying within a $\pm 2.5 \mathrm{~Hz}$ frequency band around $60 \mathrm{~Hz}$ and $80 \mathrm{~Hz}$.

- the controller should attenuate the disturbances by a minimum of $14 \mathrm{~dB}$.

- the maximum allowed amplification of the output sensitivity function is of $8 \mathrm{~dB}$.

- the effect of disturbances on the control input should be attenuated above $100 \mathrm{~Hz}$ in order to improve robustness with respect to unmodeled dynamics and nonlinear phenomena $\left(S_{u p}\left(e^{-j \omega}\right)<-20 \mathrm{~dB}, \forall \omega \in[100 \mathrm{~Hz}, 400 \mathrm{~Hz}]\right)$

- the gain of the controller should be zero at at $0 \mathrm{~Hz}$ and $0.5 f_{s}(400 \mathrm{~Hz})$ (the system has no gain at $0 \mathrm{~Hz}-$ double differentiator - and very low gain at $0.5 f_{s}$ )

It is shown in [12, Property 7, Section 3.6.1] that very accurate shaping of the output or the input sensitivity function can be obtained by the use of band-stop filters (BSF). These are IIR filters obtained from the discretization of continuoustime filters of the form

$$
F(s)=\frac{s^{2}+2 \zeta_{\text {num }} \omega_{0} s+\omega_{0}^{2}}{s^{2}+2 \zeta_{\text {den }} \omega_{0} s+\omega_{0}^{2}}
$$

using the bilinear transform $s=\frac{2}{T s} \frac{1-z^{-1}}{1+z^{-1}}$. The use of BSFs introduces an attenuation $M=20 \log \left(\frac{\zeta_{\text {num }}}{\zeta_{\text {den }}}\right)$ at the normalized discretized frequency $\omega_{d}=2 \cdot \arctan \left(\frac{\omega_{0} T_{S}}{2}\right)$. Depending on whether the filter is designed for shaping the output or the input sensitivity function, the numerator of the discretized filter is included in the fixed part of the controller denominator $H_{S_{0}}$ or numerator $H_{R_{0}}$, respectively. The filter denominator is always included in the closed loop characteristic polynomial. As such, the filter denominator influences the design of the controller indirectly in the computation of $S_{0}^{\prime}$ and $R_{0}^{\prime}$ as solutions of the Bezout equation (6). They will be used for a fine shaping of both the output and input sensitivity functions.

The steps for the design of the linear controller are: ${ }^{7}$

1) include all (stable) secondary path poles in the closed loop characteristic polynomial.

2) open the loop at $0 \mathrm{~Hz}$ and at $400 \mathrm{~Hz}$ by setting the fixed part of the controller numerator

$$
H_{R}=\left(1+q^{-1}\right) \cdot\left(1-q^{-1}\right)
$$

3) 3 BSFs on $S_{y p}$ have been used around each of the frequencies where attenuation is desired in order to assure the desired attenuation within $\pm 2.5 \mathrm{~Hz}$ (see Table I for specifications).

4) 1 BSF has been used on $S_{u p}$ to reduce its magnitude above $100 \mathrm{~Hz}$ (see Table I for specifications).

5) to improve robustness 2 complex conjugate poles have been added to the characteristic polynomial, one at $55 \mathrm{~Hz}$ and the second at $95 \mathrm{~Hz}$, both of them with 0.1 damping factor.

Table I

Band-stop filters for output and input sensitivity functions.

\begin{tabular}{|c|c|c|c|}
\cline { 2 - 4 } \multicolumn{1}{c|}{} & Frequency [Hz] & Amplification [dB] & Damping \\
\hline \multirow{4}{*}{$S_{y p}$} & 57.5 & -17 & 0.1 \\
\cline { 2 - 4 } & 59.8 & -25 & 0.5 \\
\cline { 2 - 4 } & 62 & -15 & 0.1 \\
\cline { 2 - 4 } & 77.5 & -13 & 0.05 \\
\cline { 2 - 4 } & 79.8 & -20 & 0.2 \\
\hline \hline$S_{u p}$ & 82 & -12 & 0.05 \\
\hline
\end{tabular}

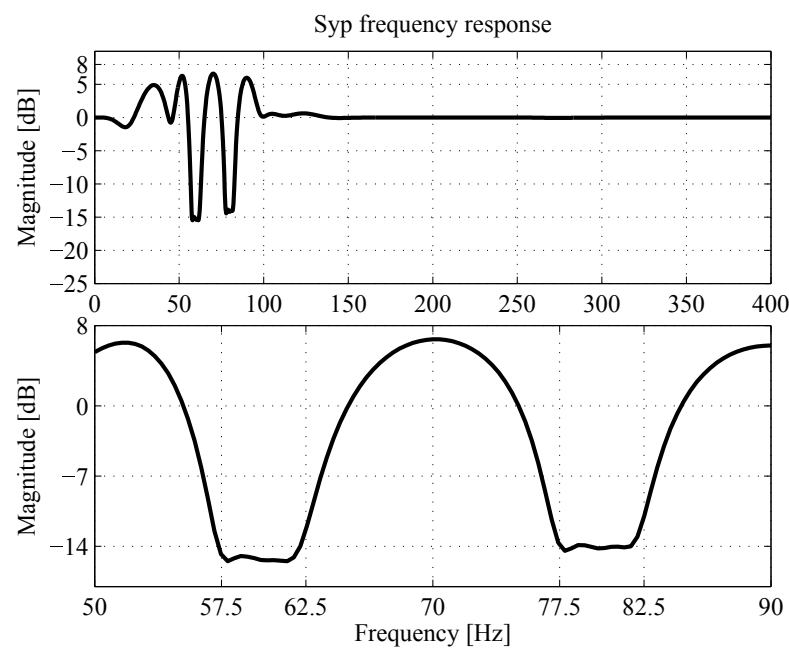

Figure 6. Output sensitivity function with the linear controller (upper figure) and zoom in the $50 \mathrm{~Hz}$ to $90 \mathrm{~Hz}$ frequency interval (lower figure).

The output and input sensitivity functions with this linear controller can be analyzed in Figs. 6 and 7, respectively. From Fig. 6, it can be observed that the desired attenuation of $14 \mathrm{~dB}$ and the maximum amplification of $8 \mathrm{~dB}$ on $S_{y p}$ are achieved. 


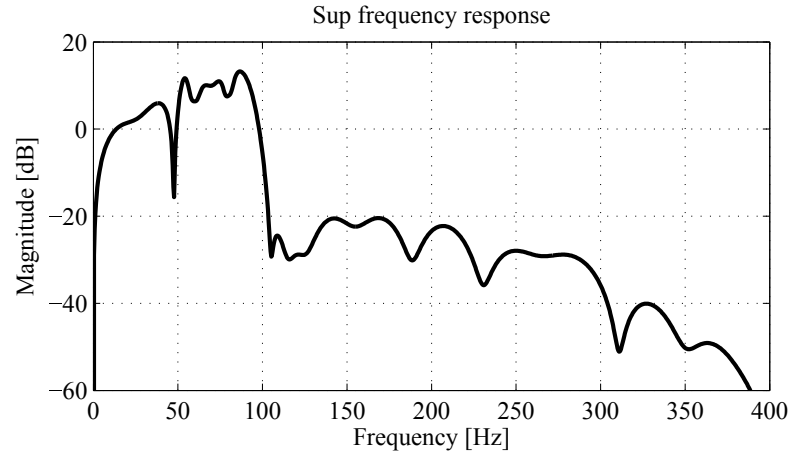

Figure 7. Input sensitivity function with the linear controller.

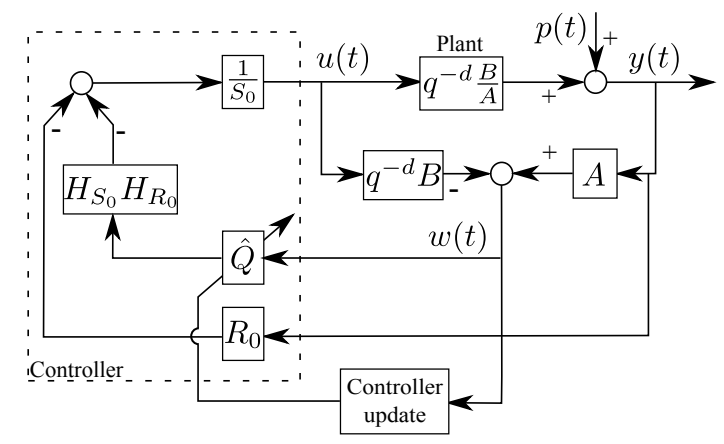

Figure 8. Direct adaptive regulation scheme for rejection of unknown disturbances using the Youla-Kučera parametrization.

\section{Adaptive Feedback Control}

This section gives a brief presentation of the direct adaptive control scheme implemented on top of the central controller. The Youla-Kučera (YK) parametrization of the controller is used (see [7]) for implementing the adaptive loop. In this context, the controller polynomials are parametrized using an finite impulse response (FIR) filter of the form

$$
Q\left(z^{-1}\right)=q_{0}+q_{1} z^{-1}+\ldots+q_{n_{Q}} z^{-n_{Q}},
$$

and the central controller polynomials given in eqs.(4) and (5) obtained as solutions of the Bezout equation (6). As such, the controller polynomials become

$$
\begin{aligned}
& R=R_{0}+A Q H_{S_{0}} H_{R_{0}}, \\
& S=S_{0}-z^{-d} B Q H_{S_{0}} H_{R_{0}} .
\end{aligned}
$$

The purpose of the central controller $\left(\frac{R_{0}}{S_{0}}\right)$ in the YoulaKučera parametrization is that of verifying stability and robustness specifications. It should be observed that the characteristic polynomial of the closed loop remains unchanged

$$
P=A S+z^{-d} B R=A S_{0}+z^{-d} B R_{0},
$$

in the presence of the $Q\left(z^{-1}\right)$ FIR filter. The role of the $Q$ filter, which will be adjusted in real time using a parameter adaptation algorithm, is to assure the rejection of unknown time-varying disturbances. The schematic representation of the closed loop with the adaptive Youla-Kučera parametrized

\footnotetext{
${ }^{7}$ The software iREG has been used for the design of this robust digital controller but the same results can be obtained using functions written in Matlab/Scilab languages (see http://www.gipsa-lab.grenoble-inp.fr/ ioandore. landau/identificationandcontrol/).
}

controller of (12) and (13) is shown in Fig. 8. Note that in Fig. $8, w(t)=A \cdot p(t)$ and as such the Youla-Kučera parametrization is a disturbance observer and $w(t)$ is feedback through the filter $Q$ (like in DOB control method [8]).

A key aspect of this methodology is the use of the IMP. It is supposed that $p(t)$ is a deterministic disturbance given by

$$
p(t)=\frac{N_{p}\left(q^{-1}\right)}{D_{p}\left(q^{-1}\right)} \cdot \delta(t),
$$

where $\delta(t)$ is a Dirac impulse and $N_{p}, D_{p}$ are coprime polynomials of degrees $n_{N_{p}}$ and $n_{D_{p}}$, respectively ${ }^{8}$. In the case of stationary narrow-band disturbances, the roots of $D_{p}\left(z^{-1}\right)$ are on the unit circle.

Internal Model Principle [5]: The effect of the disturbance (15) upon the output

$$
y(t)=\frac{A\left(q^{-1}\right) S\left(q^{-1}\right)}{P\left(q^{-1}\right)} \cdot \frac{N_{p}\left(q^{-1}\right)}{D_{p}\left(q^{-1}\right)} \cdot \delta(t),
$$

where $D_{p}\left(z^{-1}\right)$ is a polynomial with roots on the unit circle and $P\left(z^{-1}\right)$ is an asymptotically stable polynomial, converges asymptotically towards zero iff the polynomial $S\left(z^{-1}\right)$ in the RS controller has the form (based on eq. (4))

$$
S\left(z^{-1}\right)=D_{p}\left(z^{-1}\right) H_{S_{0}}\left(z^{-1}\right) S^{\prime}\left(z^{-1}\right) .
$$

Thus, the pre-specified part of $S\left(z^{-1}\right)$ should be chosen as $H_{S}\left(z^{-1}\right)=D_{p}\left(z^{-1}\right) H_{S_{0}}\left(z^{-1}\right)$ and the controller is computed solving

$$
P=A D_{p} H_{S_{0}} S^{\prime}+z^{-d} B H_{R_{0}} R^{\prime}
$$

where $P, D_{p}, A, B, H_{R_{0}}, H_{S_{0}}$ and $d$ are given ${ }^{9}$. The $Q$ polynomial allows the introduction in the controller of the model of the disturbance (i.e. if $D_{p}$ is the model of the disturbance, it exist a polynomial $Q$ of order $n_{D_{p}}-1$ such that $S$ given by (13) can be factorized as $S^{\prime} D_{p}$ - see [14]).

Assuming that the structure of the disturbance is known, i.e. $n_{D_{p}}$, the order of the $Q$ polynomial is fixed as $n_{Q}=n_{D_{p}}-1$. Let define the estimate of $Q$ at time $t$ by $\hat{Q}\left(t, q^{-1}\right)=\hat{q}_{0}(t)+$ $\hat{q}_{1}(t) q^{-1}+\ldots+\hat{q}_{n_{Q}}(t) q^{-n_{Q}}$ and the associated estimated parameter vector $\hat{\theta}(t)=\left[\begin{array}{llll}\hat{q}_{0}(t) & \hat{q}_{1}(t) & \ldots & \hat{q}_{n_{Q}}(t)\end{array}\right]^{T}$. Define the fixed parameter vector corresponding to the optimal value of the polynomial $Q$ as: $\theta=\left[\begin{array}{llll}q_{0} & q_{1} & \ldots & q_{n_{Q}}\end{array}\right]^{T}$. Denote

$$
\begin{gathered}
w(t+1)=A \cdot y(t+1)-q^{-d} B^{*} \cdot u(t), \\
w_{1}(t)=\frac{S_{0}}{P} \cdot w(t), w_{2}(t)=\frac{q^{-d} B^{*} H_{S_{0}} H_{R_{0}}}{P} \cdot w(t)
\end{gathered}
$$

and define the following observation vector

$$
\phi^{T}(t)=\left[w_{2}(t) w_{2}(t-1) \ldots w_{2}\left(t-n_{Q}\right)\right] .
$$

The a priori adaptation error can be defined as (see [13] for more details)

$$
\varepsilon^{0}(t+1)=w_{1}(t+1)-\hat{\theta}^{T}(t) \phi(t) .
$$

\footnotetext{
${ }^{8}$ Throughout the paper, $n_{X}$ denotes the degree of the polynomial $X$.

${ }^{9}$ Of course, it is assumed that $D_{p}$ and $B$ do not have common factors.
} 
For the estimation of the parameters of $\hat{Q}\left(t, q^{-1}\right)$ an 'Integral' Parameter Adaptation Algorithm (I-PAA) is used:

$$
\begin{aligned}
\hat{\theta}(t+1) & =\hat{\theta}(t)+F(t) \phi(t) \varepsilon(t+1) \\
\varepsilon(t+1) & =\frac{\varepsilon^{0}(t+1)}{1+\phi^{T}(t) F(t) \phi(t)} \\
\varepsilon^{0}(t+1) & =w_{1}(t+1)-\hat{\theta}^{T}(t) \phi(t), \\
F(t+1) & =\frac{1}{\lambda_{1}(t)}\left[F(t)-\frac{F(t) \phi(t) \phi^{T}(t) F(t)}{\frac{\lambda_{1}(t)}{\lambda_{2}(t)}+\phi^{T}(t) F(t) \phi(t)}\right], \\
1 & \geq \lambda_{1}(t)>0,0 \leq \lambda_{2}(t)<2
\end{aligned}
$$

where $\lambda_{1}(t), \lambda_{2}(t)$ allow to obtain various profiles for the evolution of the adaption gain $F(t)$ (for more details see [14]). Another more general choice of adaptation algorithm is the 'Integral+Proportional' Parameter Adaptation Algorithm (IPPAA) (see [14], [15] for more details).

At each sampling time the following procedure is applied for adaptive operation:

1) Get the measured output $y(t+1)$ and the applied control $u(t)$ to compute $w(t+1)$ using (19).

2) Compute $w_{1}(t+1)$ and $w_{2}(t)$ using (20) with $P$ given by (14).

$3)$ Estimate the $Q$-polynomial using the I-PAA of eqs. (23).

4) Compute and apply the control (see Fig. 8):

$$
S_{0}\left(q^{-1}\right) u(t+1)=-R_{0}\left(q^{-1}\right) y(t+1)-\hat{Q}\left(t+1, q^{-1}\right) w(t+1) \text {. }
$$

\section{Experimental Results}

The experimental results presented in this section are obtained using the identified model of the secondary path (see also Section II). Details on system identification and the model used throughout this section can be found on the benchmark web site at http://www.gipsa-lab.grenoble-inp.fr/ ioandore. landau/benchmark_adaptive_regulation/files/Simulator_2.zip. The secondary path model is given in the file model_sec2.mat. The orders of this system are: $n_{A}=22, n_{B}=25$, and $d=0$.

\section{A. Central Controller for Youla-Kučera Parametrization}

The design of the central controller used in the YoulaKučera parametrization is similar to the design of the robust linear controller with the exception that the BSFs on $S_{y p}$ have not been used and the resulting free roots to be assigned have been moved from 0 to 0.2 . Remark that the order of the characteristic polynomial is given by $n_{P}=n_{A}+n_{B}+n_{H_{S}}+n_{H_{R}}+d-1$ which in our case gives $22+25+0+4+0-1=50$. Given the roots already specified ( 28 as can be concluded from the design of the robust controller excepting roots given by BSFs for $S_{y p}$ ), it follows that 22 roots can be selected. These 22 auxiliary poles at 0.2 have the effect of reducing the magnitude of $S_{u p}$ above $100 \mathrm{~Hz}$. They were not used in the robust linear design.

\section{B. Single-Mode Vibration Control}

The results in this subsection are obtained by considering 2 sinusoidal disturbances with time varying frequencies on the system output. The time variations of the frequencies are obtained by using 2 independent pseudo random binary sequences (PRBS). The 2 sinusoidal disturbances vary around 60 and $80 \mathrm{~Hz}$, respectively, remaining inside the $\pm 2.5 \mathrm{~Hz}$ frequency intervals for which the robust linear controller introduces $14 \mathrm{~dB}$ of attenuation.

Note that all subsequent experiments start at 10 seconds. This period has been introduced in order to give enough time to activate the electronic boards for real time experimentation. Also, the system operates in open loop for 5 seconds (from 10 to $15 \mathrm{sec}) .{ }^{10}$ Finally, 5 seconds before the end of the experiments, the system is switched back to open loop and the system input and the disturbances are removed so that the residual forces can be compared to the system noise.

In Fig. 9, time domain experimental results are shown for the open loop, the closed loop with the linear controller and for the closed loop with the adaptive controller.

For adaptive regulation, the I-PAA is used. The order of the $Q$ polynomial has been chosen equal to 3 (4 parameters to be adapted). The initial diagonal adaptation gain matrix used is $F(0)=\alpha \cdot I$, with $\alpha=0.2$ and $I$ the identity matrix (initial trace of 0.8). A constant trace adaptation algorithm is used with constant trace of 0.8 (see [14] for further details on the choice of the adaptation gain). The evolution of the parameters of the $Q$ polynomial can be viewed in Fig. 10. As it can be observed, the vector of the estimated $Q$ parameters, $\hat{\theta}$ is initialized at zero. Once the loop is closed, the adaptive algorithm starts to adjust the parameters in order to reduce the residual force. The parameters of the Youla-Kučera filter evolve continuously during the experiments in order to adjust to the changing frequencies of the disturbances.

To compare the attenuation results of the robust linear controller with the adaptive regulator, the global attenuation is computed over the last 3 seconds of each closed loop experimentation. For the robust linear controller the global attenuation is $25.70 \mathrm{~dB}$, while in the adaptive case it is $39.68 \mathrm{~dB}$. A small additional improvement can be obtained by using the IP-PAA. ${ }^{11}$

Finally, experimental results for frequencies variations in $\pm 5 \mathrm{~Hz}$ intervals around 60 and $80 \mathrm{~Hz}$ are shown in Fig. 11 . As expected the linear controller results are not good (we are outside of the domain considered for design). The last $5 \mathrm{sec}$ without disturbance and in open loop operation are also plotted as reference. The levels of the control signal for the linear and adaptive controllers, are approximately the same: $\pm 0.14 \mathrm{~V}$ for the linear case and $\pm 0.13 \mathrm{~V}$ for the adaptive case.

\section{Two-Mode Vibrational Interference Control}

This subsection deals with the AVC of two-mode sinusoidal vibrations (vibration interference). The phenomena occurs when two tonal vibrations have very close frequencies. This phenomena is shown in the upper plot of Fig. 12 where 2

\footnotetext{
${ }^{10} \mathrm{To}$ avoid large transients when switching on the controllers, a bumpless transfer scheme from open to closed loop has been used (see also [12, Chapter $8]$ ).

${ }^{11}$ Global attenuation in decibels $(\mathrm{dB})$ is computed as $-20 \log _{10}\left(\frac{\operatorname{var}\left(y_{C L}\right)}{\operatorname{var}\left(y_{O L}\right)}\right)$, where $y_{O L}$ denotes residual acceleration in open loop and $y_{C L}$ is the closed loop residual acceleration.
} 

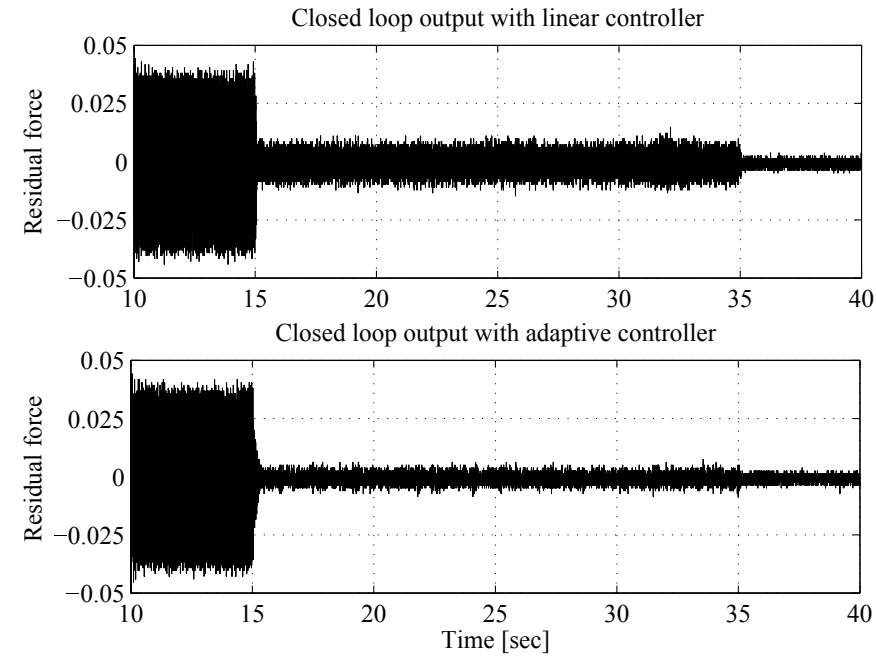

Figure 9. Residual force in closed loop with linear controller (upper plot) and with adaptive controller (lower plot). The experiments are started in open loop for 5 seconds. Range of frequency variation: $\pm 2.5 \mathrm{~Hz}$.

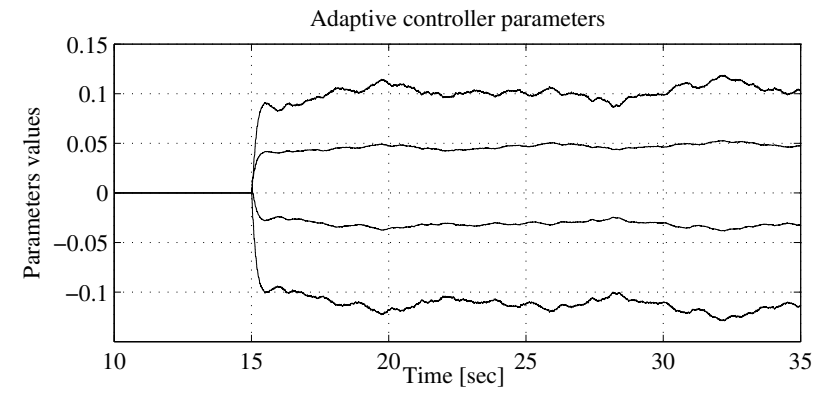

Figure 10. Evolution of the Q-parameters.

pairs of neighboring sinusoidal disturbances are introduced, one pair around $60 \mathrm{~Hz}$ (at 59.9 and $60.1 \mathrm{~Hz}$ ) and the second around $80 \mathrm{~Hz}$ (at 79.9 and $80.1 \mathrm{~Hz}$ ). The same robust linear controller as described earlier can be used as its attenuation frequency band is large enough to accommodate the neighboring disturbances.
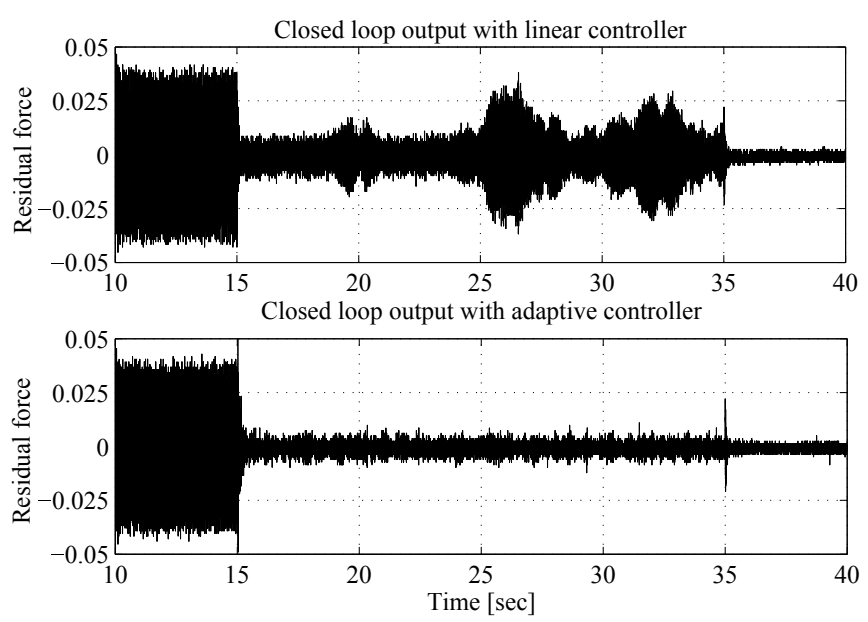

Figure 11. Residual force in closed loop with linear controller (upper plot) and with adaptive controller (lower plot). The experiments are started in open loop for 5 seconds. Range of frequency variation: $\pm 5 \mathrm{~Hz}$.
For adaptive regulation, the I-PAA has been used with an initial diagonal adaptation gain matrix $F(0)=\alpha \cdot I$, with $\alpha=0.2$ and $I$ the identity matrix (initial trace of 0.8 ), and a decreasing gain followed by constant trace adaptation. The constant trace is chosen equal to 0.02 . The number of parameters for the $Q$ polynomial is also equal to 4 (order equal to 3). Augmenting the order of the polynomial $Q$ to 7 (8 parameters - two for each sinusoidal disturbance) does not improve the performance (probably because the frequencies of the pair of sines are too close). Time domain results are shown in Figs. 12. The global attenuation for the robust linear controller is $27.50 \mathrm{~dB}$ and for the adaptive controller is $45.59 \mathrm{~dB}$.

Power spectral densities (PSD) estimates of the two control schemes are given in Fig. 13. It can be observed that the attenuation introduced by the robust linear controller in the desired frequency zone is equal to $14 \mathrm{~dB}$ which is coherent with the design done in Section IV. The adaptive regulator has better attenuation of disturbances and also does not amplify at other frequencies more than the linear controller.
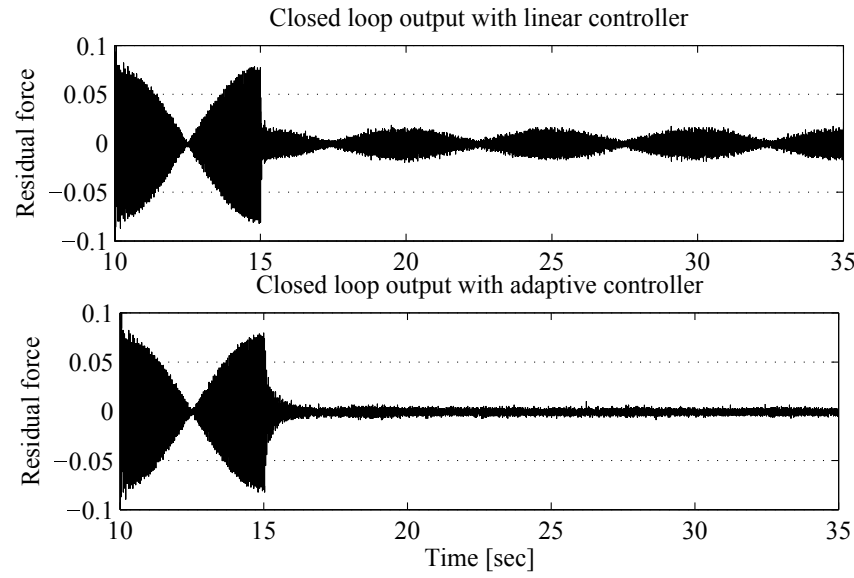

Figure 12. Residual force in closed loop with linear controller (upper plot) and with adaptive controller (lower plot). The loop is closed at $\mathrm{t}=15 \mathrm{sec}$

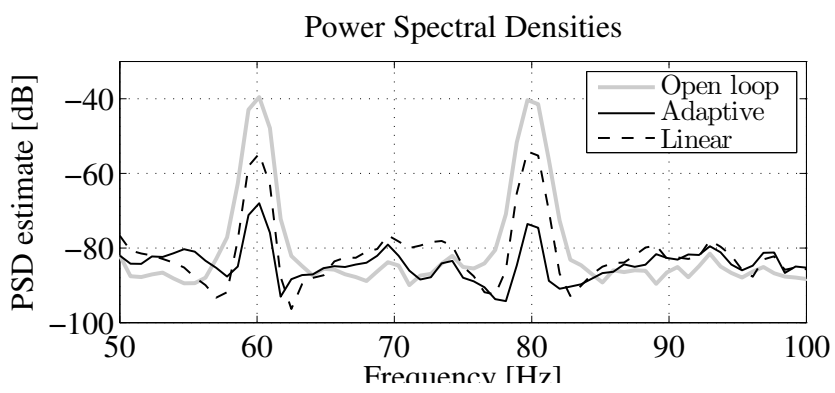

Figure 13. Power spectral densities of the open loop (grey solid line), robust linear (dashed line), and adaptive (solid black line) controllers. Zoom between 50 and $100 \mathrm{~Hz}$.

Adaptation capabilities are tested and the results are compared to the linear robust controller in Fig. 14. In this figure, all 4 sinusoidal disturbances are modified at 35 seconds by adding $5 \mathrm{~Hz}$ to their frequencies. The constant trace is increased to 0.1 . As such the new disturbance frequencies are centered around $65 \mathrm{~Hz}(64.9$ and $65.1 \mathrm{~Hz})$ and $85 \mathrm{~Hz}$ (84.9 and $85.1 \mathrm{~Hz}$ ). As expected the linear robust controller 
fails to provide an acceptable attenuation. The adaptation transient is about $1.5 \mathrm{sec}$. The input signal for both linear control and adaptive control is shown in Figure 15. Their level is comparable in the nominal situation but when the frequencies changes the level of the control signal for the adaptive controller is lower.
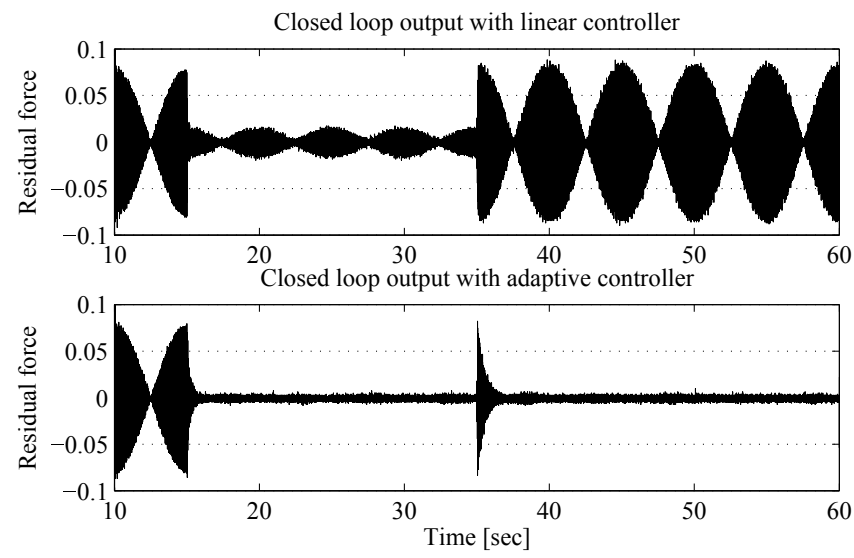

Figure 14. Vibrational interference. Residual force with step frequency changes $(+5 \mathrm{~Hz}$ at $35 \mathrm{sec})$ in closed loop with linear controller (upper plot) and with adaptive controller (lower plot). The system is in open loop until $\mathrm{t}=15 \mathrm{sec}$.
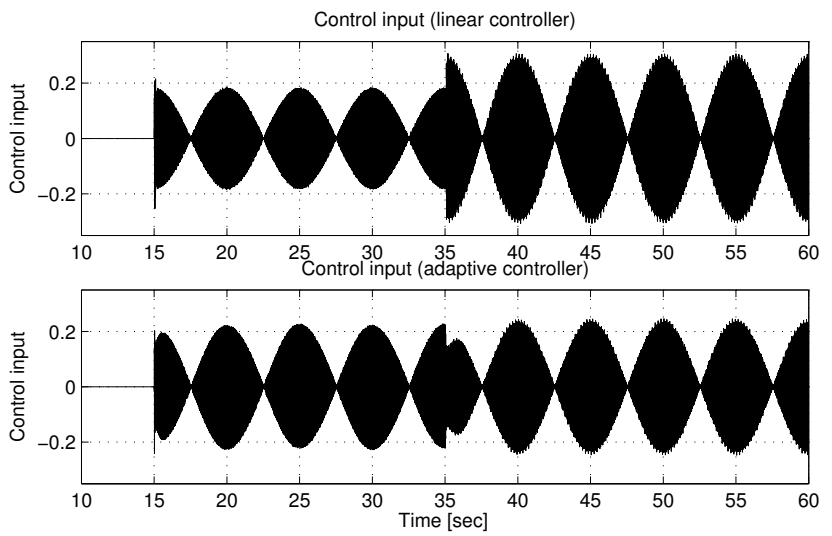

Figure 15. Control signals for step frequency changes $(+5 \mathrm{~Hz})$ experiment: linear controller (upper plot) and adaptive controller (lower plot).

\section{Conclusion}

It was shown in this paper that provided that the region of variation of the unknown multiple tonal vibrations is limited, efficient robust active compensation can be achieved with a properly designed linear controller. However, adding an adaptive loop enhances drastically the performance. The use of the adaptive approach allows to achieve the same level of performance even if the variations of the frequencies of the vibrations go beyond the initial specified frequency region. In this new context the performance of the robust controller are unsatisfactory.

\section{References}

[1] T. Marcos, "The straight attraction," Motion control, vol. 13, pp. 29-33, 2000.
[2] S. Li, J. Qiu, J. Li, H. Ji, and K. Zhu, "Multi-modal vibration control using amended disturbance observer compensation," Control Theory Applications, IET, vol. 6, no. 1, pp. 72-83, January 2012.

[3] S. Li, J. Li, Y. Mo, and R. Zhao, "Composite multi-modal vibration control for a stiffened plate using non-collocated acceleration sensor and piezoelectric actuator," Smart Materials and Structures, vol. 23, no. 1, p. 015006, 2014.

[4] S. Elliott and T. Sutton, "Performance of feedforward and feedback systems for active control," IEEE Speech Audio Proces., vol. 4, no. 3, pp. $214-223$, may 1996.

[5] B. Francis and W. Wonham, "The internal model principle of control theory," Automatica, vol. 12, no. 5, pp. 457 - 465, 1976.

[6] I. D. Landau, A. Castellanos Silva, T.-B. Airimițoaie, G. Buche, and M. Noé, "Benchmark on adaptive regulation: rejection of unknown/timevarying multiple narrow band disturbances," European Journal of Control, vol. 19, no. 4, pp. 237 - 252, 2013.

[7] B. Anderson, "From Youla-Kucera to identification, adaptive and nonlinear control," Automatica, vol. 34, pp. 1485-1506, 1998.

[8] S. Li, J. Qiu, H. Ji, K. Zhu, and J. Li, "Piezoelectric vibration control for all-clamped panel using dob-based optimal control," Mechatronics, vol. 21, no. 7, pp. $1213-1221,2011$

[9] S. Li, J. Li, and Y. Mo, "Piezoelectric Multimode Vibration Control for Stiffened Plate Using ADRC-Based Acceleration Compensation," IEEE Trans. Ind. Electron., vol. 61, no. 12, pp. 6892-6902, 2014.

[10] J. C. Carmona and V. M. Alvarado, "Active noise control of a duct using robust control theory," IEEE Trans. Control Syst. Technol., vol. 8, no. 6, pp. 930-938, 2000.

[11] J. Langer and I. D. Landau, "Combined pole placement/sensitivity function shaping method using convex optimization criteria," Automatica, vol. 35 , no. 6, pp. 1111-1120, 1999.

[12] I. D. Landau and G. Zito, Digital Control Systems - Design, Identification and Implementation. London: Springer, 2005.

[13] I. D. Landau, A. Constantinescu, and D. Rey, "Adaptive narrow band disturbance rejection applied to an active suspension - an internal model principle approach," Automatica, vol. 41, no. 4, pp. 563-574, 2005.

[14] I. D. Landau, R. Lozano, M. M'Saad, and A. Karimi, Adaptive control, 2nd ed. London: Springer, 2011.

[15] T.-B. Airimițoaie and I. D. Landau, "Improving adaptive feedforward vibration compensation by using "Integral+Proportional" adaptation," Automatica, vol. 49, no. 5, pp. 1501 - 1505, 2013.

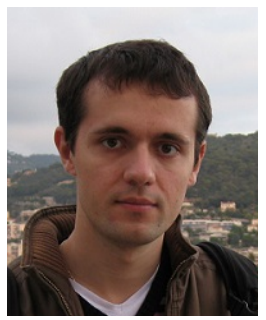

Tudor-Bogdan Airimitoaie was born in Suceava, Romania, in 1983. He received an Automatic Control Engineering Degree from the University "Politehnica" of Bucharest, Romania in 2008 and a $\mathrm{PhD}$ Degree from the University of Grenoble, France and the University "Politehnica" of Bucharest, Romania in 2012. Currently he is with the University of Bordeaux, France. His research interests are in the fields of robust adaptive active control, identification, fractional order systems, and flatness.

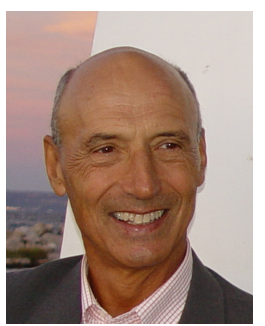

Ioan Doré Landau is Emeritus Research Director at C.N.R.S. (National Center for Scientific Research) at the GIPSA-LAB (CNRS/INPG), Control Department in Grenoble. His research interests encompass theory and applications in system identification, adaptive control, robust digital control and nonlinear systems. He has authored and co-authored over 200 papers on these subjects. He is the author and coauthor of several books including: Adaptive Control - The Model Reference Approach (Dekker 1979), Digital Control Systems (Springer 2005), Adaptive Control (Springer 2011, 2nd edition). Dr. Landau received the Rufus Oldenburger Medal 2000 from the American Society of Mechanical Engineers. He is "Doctor Honoris Causa" of the Université Catholique de Louvain-la-Neuve. He received also the CNRS Silver Medal and the Price Montpetit from the French Academy of Science. 